\title{
OBSERVATORIO
}

\section{TENDENCIAS TECNOLÓGICAS EN INTERNET: HACIA UN CAMBIO DE PARADIGMA}

\section{Internet technology trends: Towards a paradigm shift}

\section{Jorge Serrano-Cobos}

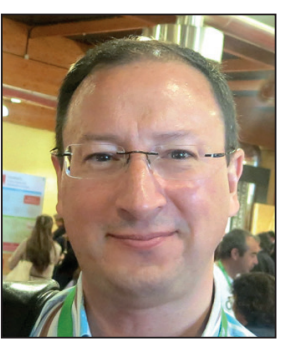

Jorge Serrano-Cobos, profesor asociado de la Universidad Politécnica de Valencia (masters Mugi y Calsi) y profesor invitado en diversas universidades, es miembro fundador del Grupo de Investigación Trademetrics. Es director de innovación en Metric Salad, una start-up especializada en cibermetría, analítica digital, SEO, social media analytics, social network analysis, minería web y visualización de datos.

http://trademetrics.upv.es

http://orcid.org/0000-0002-4394-4883

Universidad Politécnica de Valencia Departamento de Comunicación Audiovisual, Documentación e Historia del Arte Grupo de investigación Trademetrics Camí de Vera, s/n. 46022 Valencia, España jorserc2@upv.es

\section{Resumen}

Se describen las innovaciones y tendencias tecnológicas de 2016, y se hace un análisis holístico de hacia dónde puede llevar internet a la sociedad actual. Se toma el punto de vista de la relación entre usuarios/clientes e instituciones/marcas, a la luz de multitud de ejemplos reales. Los ejes sobre los que está girando el nuevo paradigma de internet (con especial atención al marketing digital, la comunicación, y la gestión de la información) son, entre otros: auge de la inteligencia artificial, inmediatez, personalización, big data, internet de las cosas, y variedad de tipos y canales de interacción.

\section{Palabras clave}

Internet; Tecnologías de la información; Tendencias; Innovación; Transformación digital; Personalización; Interacción; Inteligencia artificial; Big data; Datos masivos; Interacción hombre-máquina; Marketing.

\section{Abstract}

Innovations and technological trends in 2016 are discussed along with a holistic analysis of where the Internet can may lead today's society. The relationship between users/clients and institutions/brands is considered. The paradigm for the internet has shifted; internet marketing, communication, and information management revolve around the topics of artificial intelligence, immediacy, personalization, big data, internet of things, and multiple forms of interaction.

\section{Keywords}

Internet; Information technologies; Trends; Innovation; Digital transformation; Personalization; Interaction; Artificial intelligence; Big data; Human computer interaction; Marketing.

Serrano-Cobos, Jorge (2016). "Tendencias tecnológicas en internet: hacia un cambio de paradigma". El profesional de la información, v. 25, n. 6, pp. 843-850.

https://doi.org/10.3145/epi.2016.nov.01

Aunque la idea de "paradigma" se circunscribía en origen a la ciencia como "teoría o conjunto de teorías cuyo núcleo central se acepta sin cuestionar y que suministra la base y modelo para resolver problemas y avanzar en el conocimiento" (RAE, 2016), el concepto se suele usar hoy en otros ámbitos no científicos.

Así, cuando se habla de "cambio de paradigma" se sobreen- tiende que ese cambio, sea en el contexto que sea, refleja una revolución profunda en un modelo preconcebido.

Cabe preguntarse si internet está provocando un cambio de paradigma, entendiendo internet como esa mega-entidad que es algo más que la suma de una red de nodos, servidores, dominios, instituciones... Y como todo cambio, si no es una moda hay que tomárselo en serio para no quedarse atrás. 
¿Qué está cambiando en estos últimos tiempos para intuir este "cambio de paradigma" motivado por internet $y$ en lo que representa para las vidas de los ciudadanos?

Analicemos algunas de las principales tendencias, no sólo tecnológicas, que se están percibiendo, lo que nos permitirá completar una visión global de lo que está sucediendo.

\section{¿Ya estamos todos en internet?}

Según el informe de Mary Meeker Internet trends 2016 (Meeker, 2016), los grandes números relacionados con el uso de internet se están ralentizando, como si todos los usuarios que hoy pudieran estar en internet, ya lo estén (figura 1).

\section{Transformación digital}

La problemática, los inconvenientes y las ventajas del cambio de los procedimientos manuales a los informáticos o digitales (automatización, informatización, digitalización) no es nueva pues ya se estudiaba a principios de los 80 (Capgemini, 2012), pero en los últimos años ha ido ganando volumen debido a que:

- la adopción de la tecnología necesaria para cada actividad está aumentando gracias al abaratamiento de costes, y principalmente gracias al software open source y al modelo de funcionamiento Saas (software as a service y cloud computing): no hace falta instalar los programas en nuestro ordenador local sino que se usan unos instalados en un servidor externo o remoto (en la nube), compartidos con otros usuarios;

- internet está entrando en todos los procesos y aspectos de los negocios, ya sean intensivos en tecnología y conectividad, o no.

\section{¿Qué está cambiando para intuir un cambio de paradigma en internet y en lo que representa para las vidas de los ciudadanos?}

Tras haber ido ganando en competencia, la capacidad transformadora de la alfabetización digital ha llevado a los usuarios/clientes, a los proveedores, a las máquinas, a vivir prácticamente conectados todo el día. Así, los negocios y entidades públicas que antes sólo vivían limitados a su entorno local, ahora deben repensar sus modelos de negocio y sus procesos para integrar tanto procesos locales como globales, y tanto offline como online, yendo más allá de convertirse en entidades "libres de papel", lo que no es poco (Westerman; Bonnet; McAfee, 2014). En la figura 2 puede verse un diagrama de los elementos que conforman el actual mundo internet clasificados en procesos, productos y sistemas en los que intervienen más directamente las personas.

\section{En 2019 el vídeo representará el 80\% del tráfico mundial de internet}

\section{Vídeo en vivo}

Cuando YouTube nació en 2004 no era fácil sospechar que iba a revolucionar la gestión de contenidos tanto como lo ha hecho, puesto que su modelo de negocio, más caro de mantener cuanta más información y más visitas recibía, hacía presumible pensar que no iba a durar mucho.

La entrada de Google -que compró YouTube en 2006 por 1.650 millones de US\$- lo cambió todo, y hoy en día es indiscutible la presencia central de YouTube en las vidas de los internautas. Según la propia empresa, este medio social de vídeo tiene más de mil millones de usuarios, casi un tercio de todas las personas usuarias de internet (YouTube, 2015).

Mientras, Facebook ha entrado en el juego, buscando quitar audiencia a Google/YouTube. De acuerdo con un informe de Cisco (Cisco, 2015), en 2019 el vídeo representará el $80 \%$ del tráfico mundial de internet. La evo- 
lución de esta tendencia es el vídeo en vivo, con plataformas como Periscope y Meerkat, que suman millones de emisores de vídeos diarios.

\section{Chatbots}

La interacción por voz mediante el móvil nos lleva a la evolución de la búsqueda, hablando en vez de escribiendo (Siri, Cortana), pero junto a ella ha aparecido otro software que llevaba tiempo siendo usado sólo por unos pocos servicios a clientes:

Un chatbot es un software que interactúa con el usuario mediante una interfaz de conversación, diseñado para simular una conversación inteligente sin un ser humano presente. Ofrecen una interesante flexibilidad con el fin de automatizar tareas, y ayudar en la recuperación de datos. Se están convirtiendo en una forma vital para mejorar la experiencia del consumidor con un servicio $365 \times 24 \times 7$. En Facebook Messenger los hay a miles (Rosenberg, 2016), para muy diferentes tareas, como ir filtrando productos de forma facetada pero mediante una conversación, promocionar un curriculum vitae, y hasta se pueden construir ya sin programación:

https://www.motion.ai

Un chatbot es un software que interactúa con el usuario mediante una interfaz de conversación, diseñado para simular una conversación inteligente sin un ser humano presente

La pregunta es qué influencia tendrán en la actual forma de buscar y gestionar la información. Existen evidencias de que ya planteamos nuestras búsquedas de forma diferente (usando lenguaje natural en los buscadores) y adaptándonos a los chatbots y en general guiados o conducidos por las interfaces.

\section{Contenido social que desaparece}

La irrupción de Snapchat incluyó un elemento de innovación al permitir que el contenido audiovisual desapareciera tras un breve tiempo de exposición. Esa inmediatez impone nuevos hábitos y nuevas reglas de juego, lo que a buen seguro hace zozobrar muy distintos paradigmas:
- los de recuperación de información: no se puede recuperar un contenido que ya no está;

- los de marketing: cómo hacer branding cuando el contenido que lo soporta muere.

La inmediatez de Snapchat impone nuevos hábitos y nuevas reglas de juego y afecta a distintos paradigmas de recuperación de la información y de marketing

\section{Concentración de hubs sociales}

Como ya decía Tim Wu (Wu, 2010), cualquier industria en evolución se mueve desde un modelo tipo "frontera del oeste salvaje" en el que aparecen muchas opciones aisladas como setas, a un modelo de grupo más centralizado y concentrado de los jugadores, lo que no deja de ser un corolario del efecto Mateo: "quien más tiene más recibe" (Merton, 1968). Cuando se empiezan a concentrar unos cuantos, inmediatamente atraen a otros, que dejan de estar aislados

\section{Automatización del marketing}

Es un concepto muy antiguo, y en la era de internet comen- 


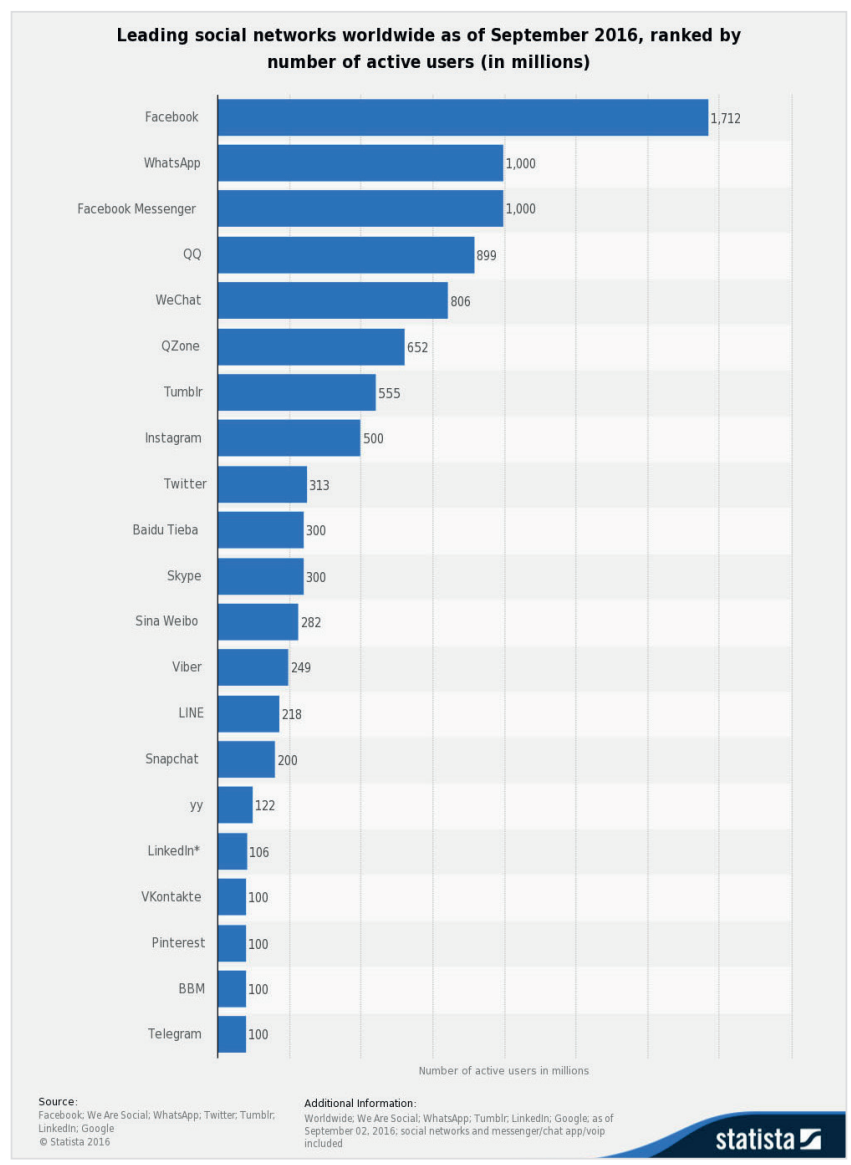

Figura 3. Número de usuarios activos por redes sociales https://www.statista.com/statistics/272014/global-social-networksranked-by-number-of-users

zó con los autorespondedores de e-mail marketing: por ejemplo, un usuario se daba de alta en un servidor a través de un formulario y el sistema contestaba, pero de paso hacía preguntas, ¿̇quiere que le llamemos? o ¿quiere ver otros productos similares?

Actualmente la innovación está en la integración de esa automatización con diversas aplicaciones y fuentes de datos, que se van haciendo más asequibles al bolsillo de las pymes, y su uso se va extendiendo para hacer más confortable la vida del pequeño profesional del marketing (Bullas, 2016).

Se puede encontrar diversas técnicas:

- remarketing (colocación de cookies en el ordenador del usuario, que permiten a la empresa enviarle posteriormente anuncios -la defensa contra esto es navegar de incógnito o buscar la cookie del ordenador y borrarla); reposting de posts antiguos (dado lo efímero y fugaz de la comunicación actual, en la que prima la inmediatez, se pueden volver a postear automáticamente contenidos antiguos que no se hayan vuelto obsoletos, artículos que no dependan del momento);

- captación de leads a través de los botones call to action (llamadas a la acción) que un usuario puede clicar para confirmar un dato, comprar, enviar su mail, o dar un "me gusta" en una página de la empresa. Un lead es una traza de cliente potencial, de un usuario que ya ha dejado de ser anónimo por haber dado ese primer clic;

- pop-ups que aparecen debido a diversos triggers o disparadores que actúan según unos parámetros marcados con anterioridad. Su misión es aumentar de forma escalable (con menor esfuerzo humano) la ratio de conversión de visitas en ventas, por ejemplo, en reservas de plazas hoteleras, descargas de documentos, registros en plataformas, boletines, o compra directa online (figura 4).

\section{Bloqueo de publicidad}

En 2015 los programas de bloqueo de publicidad crecieron un $48 \%$ en EUA y un $35 \%$ en Europa (Manson; Whatley, 2015), sin que por ello disminuya el volumen de publicidad en internet. Esto ha llevado a muchos profesionales de marketing a invertir más esfuerzo si cabe en publicidad nativa: por ejemplo, haciendo publirreportajes para el medio o la marca, pero intentando que parezcan hechos por un bloguero, adaptándose al perfil del público que visita ese blog) y en inbound marketing (marketing haciendo buenos contenidos que atraigan a los clientes. Cada vez se producen más contenidos de todo tipo (textos, imágenes, vídeos), lo que lleva a saturar aún más internet de contenido enfocado al SEO (contenidos que intentan que los buscadores les den prioridad para aparecer en la primera pantalla de resultados). Como hay mucha más competición entre contenidos se necesita más esfuerzo SEO. 


\section{Personalización basada en la identidad}

El identity-based pay-per-click marketing en el sector de la publicidad interactiva consiste en anuncios que surgen al identificarse dónde ha estado antes un usuario o al conocer sus intereses. Se ofrece así contenido relevante con un ritmo de conversión a ventas mucho más alto.

Si antes la publicidad se podía orientar por palabra clave (AdWords), o por temáticas (AdSense), ahora se puede publicitar a personas con anuncios muy específicos, incluso en base a su número de teléfono y correo electrónico (Kim, 2015). Mediante machine learning (aprendizaje de máquinas) se crean grupos homogéneos de identidades que sirven de entrenamiento para los algoritmos que deciden a quién publicitarle qué contenido.

\section{Influencer marketing}

A medida que aumenta el ruido en internet, y es más difícil llegar al cliente objetivo, el análisis de la influencia se hace vital. Las marcas intentan descubrir a los líderes de opinión (influencers) y por ello pagan para llegar a sus admiradores y seguidores, en lugar de que sea al contrario. Antes un "admirador" o fan hacía esfuerzos para obtener información de sus productos o servicios preferidos. Ahora, esos seguidores si son "estrella" y tienen audiencia puede que se profesionalicen y cobren de las marcas, trabajan de fan.

\section{Economía de la confianza y economía social}

En el modelo de la web 2.0 las marcas tratan a los usuarios como iguales para mejorar su transparencia y ganar su confianza (The economist, 2013), y son valoradas por ellos (contabilizándose más de 800 millones de quejas sociales sólo en EUA en 2014, según Koetsier, 2014). Hoy en día ese modelo ha evolucionado y es más complejo (Botsman, 2012). Un buen ejemplo es Uber, cuyo portal puntúa la confianza en el conductor, pero también en el pasajero (figura 5).

\section{Precios dinámicos}

La inmediatez en los precios es otra tendencia. Un ejemplo de ello es nuevamente Uber, que puede variar un precio en minutos en función de la oferta y la demanda (Carat, 2015). Hay empresas como Miderest, que realizan inteligencia competitiva de precios casi en tiempo real, lo que afecta a muchos aspectos del posicionamiento, pues la percepción "marca versus precio" puede cambiar en minutos.

\section{Inteligencia artificial y machine learning}

Según Cisco, el tráfico internet mundial anual superará el umbral del zetabyte ( $10^{21}$ ó 1 seguido de 21 ceros) en 2016. Buena parte de esos datos acaban en manos de unos pocos, que usan tecnología de deep learning (aprendizaje profundo) de muchas formas. Por ejemplo, podemos verlo en:

- sugerencias de Facebook de contenido patrocinado o no;

- coches de conducción automática;

- algoritmos de relación entre noticias, temas de tendencias o personas;

- adecuación entre la oferta de la empresa y el candidato en LinkedIn;

- optimización de la experiencia de usuario en Twitter mostrando contenidos servidos vía algoritmo, no (o no sólo) por orden cronológico.

\section{La influencia de la inteligencia artificial en internet hoy ya es fundamental}

Su influencia en internet hoy ya es fundamental: por ejemplo, con la sustitución de un equipo completo de personas en Facebook, para que un algoritmo decida editorialmente qué noticias son importantes de manera automática, supervisado por ingenieros de la compañía (Dans, 2016). Google está usándolo ya extensivamente para personalizar la experiencia de búsqueda y dar respuestas al público en forma de Google Cards, y no sólo listas de enlaces, lo que contrasta con el paradigma de recuperación basado en el contenido (figura 6). Pero pueden generar problemas también, como el algoritmo usado en EUA para predecir el riesgo de criminalidad de un acusado, y que puede estar sesgado racialmente (Angwin et al., 2016). Al acumularse unas primeras denuncias de personas de color el sistema prejuzgaba que todo sería así.

Algo parecido ocurrió en determinado momento con Library Thing, que al ser muy visitado por frikis empezó a recomendar libros sólo para ese tipo de lector.

https://www.librarything.com

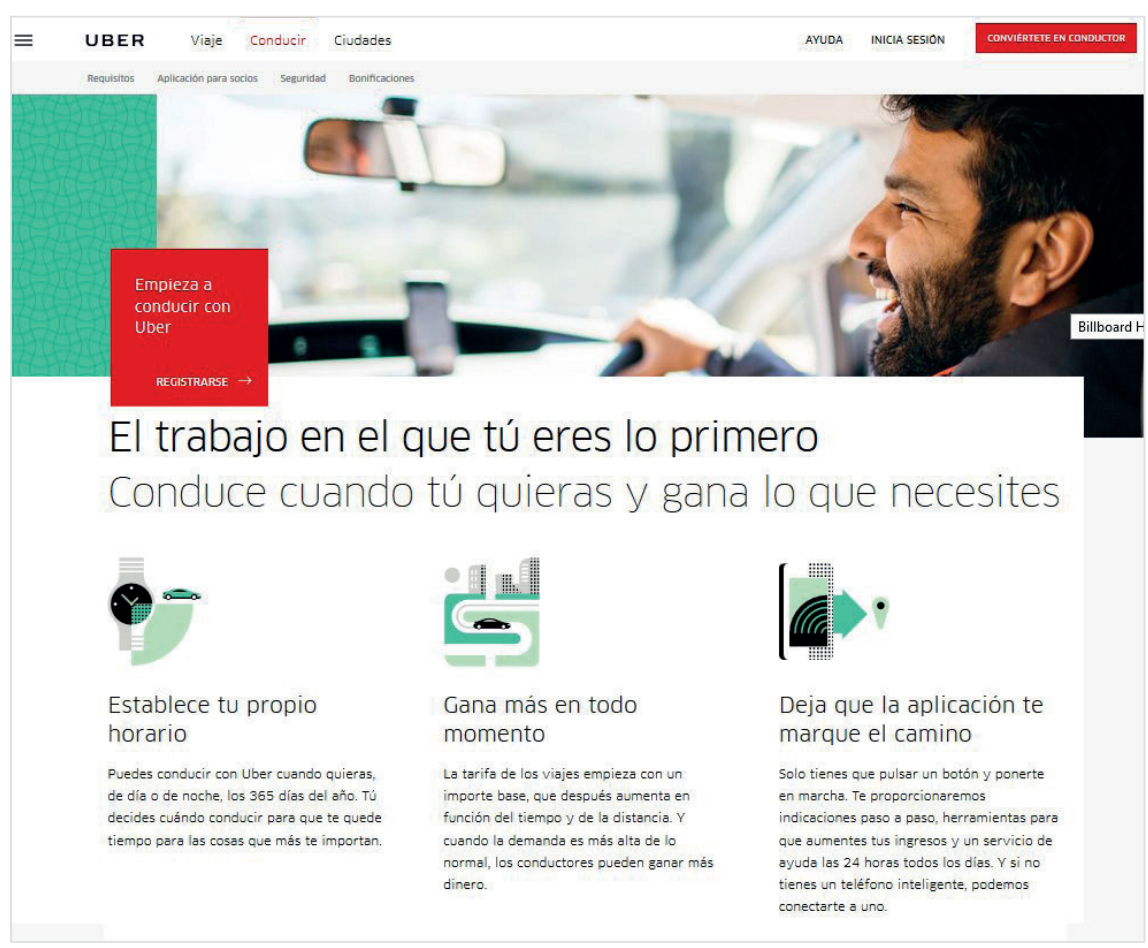

Figura 5. https://www.uber.com 
Biblioteca Nacional de España unww.bne.es/ La BNE es la institución bibliotecaria superior del Estado,
Patrimonio Bibliográfico de España. Colecciones, servicios Cotálogos Catálogos. Compartir en Twitter Colecciones Archivo de la Bibliotece - Fotografte Archivo de la Bib Hemeroteca Digital La Hemeroteca Digital forma parte de proyecto Biblioteca Digith Más resultados de bne.es ,

Biblioteca Nacional de España - Wikipedia, la enciclopedia libre https:/les.wikipedia.org/wiki/Biblioteca_Nacional_de_España perarado del depósito del

Biblioteca Nacional de España | Facebook Www.facebook.com , Places , Madrid, Spain , Library -
Paseo de Recoletos 20 . Madrid 28001 ... Biblioteca Nacional de España added 18 new photos to the album: 27 de septiembre: Dla Mundial del Turismo
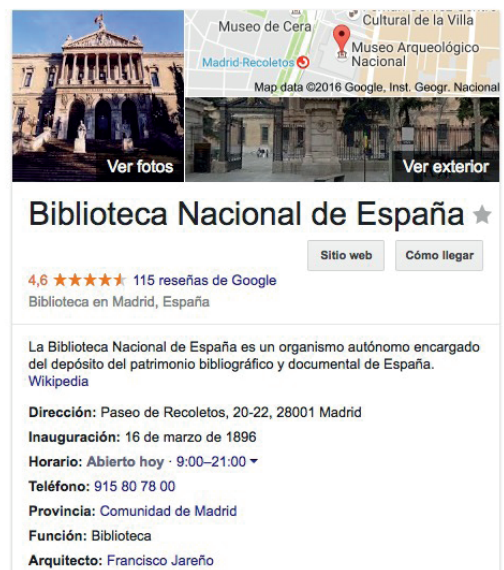

Internet of things (IOT)

Internet de las cosas es en sí una arquitectura de información que facilita el intercambio de bienes y servicios. La Comisión Europea está intentando examinar los aspectos relevantes de un posible régimen de gobernanza de la loT (Weber, 2013), fomentando estándares y buenas prácticas (como las normas ISO sobre smart cities y otras), pues la barrera entre el on (internet) y el off (las cosas, la realidad) se diluye. Existen anuncios electrónicos en la calle que muestran una publicidad $u$ otra según los móviles que circulan cerca del mismo. Las ciudades inteligentes deberían dar paso a provincias inteligentes, países inteligentes https://goo.gl/1xODYx

\section{Contenido inteligente}

La personalización y la inteligencia artificial llegan a todos los ámbitos de internet, y así la última tendencia de los sistemas de gestión de contenido es gestionar la maquetación de la información en forma de bloques en lugar de mediante plantillas. Cada bloque puede aparecer o no, o conllevar que dentro del bloque aparezca una información u otra, en función de ciertos parámetros, ciertos triggers o en función de lo que el sistema haya aprendido de la interacción del usuario o del grupo o cluster en el que su identidad haya sido incluida. De nuevo, el concepto tiene al menos dos décadas de vida, pero entonces se denominaba adaptive hipermedia (Brusilovsky, 1996) y se circunscribía al entorno científico. Hoy la tecnología necesaria se está "comoditizando", de forma que es más fácil (y rentable) desarrollar sistemas que antes eran prohibitivos.

\section{Multicanalidad (dialnet)}

Si el consumidor utiliza cualquier canal a su alcance para obtener lo que busca y es mucho más exigente y está más informado, las marcas deben aumentar la variedad de canales mediante los que se comunican con sus clientes. Gracias a la omnicanalidad (Ros-Selva, 2015), las marcas se pueden comunicar interrelacionando diversos canales entre sí y sus clientes pueden no ser conscientes de que están usando un canal u otro mientras siguen pegados a la marca.

\section{Parcelación (walled gardening)}

Los sitios web, y especialmente las apps, buscan parcelar internet, consiguiendo que cada aplicación conlleve su propio universo, del que no salgan sus usuarios, creando una contradicción (Carat, 2015): fomentando una apertura entre usuarios (al mezclarse usuarios de distintos países y culturas) pero poniendo barreras a un cruce entre contenidos de diversas plataformas. y/o continentes inteligentes, pero por ahora no dejan de ser islas inteligentes. Falta interoperabilidad entre esas islas, de manera que se pueda actuar u operar siempre igual entre sistemas. Así si una ciudad hace un sistema de información turística, debería ser trasvasable a cualquier otra ciudad, de manera que la gente no tenga que estar cambiando de sistema cuando viaja. En otros aspectos el cruce de datos y acciones multiplica las posibilidades de transformación digital, nuevos negocios. Desde la industria 4.0 (o de la cuarta revolución industrial, que integra la fabricación avanzada, robótica, inteligencia artificial, loT...), hasta las wearable technologies (tecnologías vestibles) las posibilidades del concepto de interacción también se expanden: interacción por voz, por movimiento, por temperatura corporal, por ritmo cardíaco...

Las posibilidades del concepto de interacción también se expanden: interacción por voz, por movimiento, por temperatura corporal, por ritmo cardiaco...

\section{Realidad virtual ¿Por qué todo?}

Es quizá una de las grandes incógnitas de la década. Actualmente se está viviendo una fase de aprendizaje entre la población (Carson, 2015), pero es una de las interacciones que de momento parecen estar teniendo más rechazo culturalmente. La razón es que la cacharrería (móviles, gafas, cascos...) ha sido muy cara y con mala calidad visual que provoca mareos y dolor cabeza. Existen ya modelos más manejables y usables. Como en casos anteriores (recuérdese la evolución de los móviles), harán falta más "comoditización" y precios asequibles, pero las aplicaciones prácticas (serias y lúdicas) están ahí, por lo que su uso en masa tarde o temprano llegará. 


\section{Ordenador cuántico}

La ley de Moore está a punto de expirar, como indica la Semiconductor Industry Association (Rosso, 2016) pues es imposible seguir encogiendo los chips al ritmo de antes. Pero hay otras alternativas en marcha, como un rediseño en su arquitectura, o como Google está investigando, un chip cuántico capaz de superar el rendimiento de los ordenadores convencionales en determinados tipos de cálculo (Condliffe, 2016). Se espera que sea capaz de llegar a los 50 qubits (bits cuánticos) a finales de 2017. Su aplicación directa a cualquier aspecto de la vida (optimización de resultados de buscadores, datos masivos, internet de las cosas...) nos lleva a más inmediatez si cabe.

Internet es continente y contenido de una economía social que necesita más que nunca de la transparencia

\section{Conclusiones}

Los datos masivos, su explotación vía inteligencia artificial, y la supresión de fronteras entre canales, dispositivos y tipos de interacción, nos hacen visualizar una internet que lo llena y lo ocupa todo. La inmediatez y la personalización convierten al usuario en una fuente de datos cambiante pero susceptible de ser cambiada -por efecto de los chatbots, la interacción por voz, o porque Google nos propone determinadas cadenas de términos que nos guían pero al mismo tiempo nos los impone-. Además, la capacidad de cambio de conducta por parte de aquellos que tienen el poder de transformar nuestra experiencia de interacción, es constantemente auditada por una sociedad en la que todo son usuarios y marcas. Ponemos críticas en servicios (TrypAdvisor, Uber, etc.) y al mismo tiempo podemos ser criticados.

Internet es continente y contenido de una economía social que necesita más que nunca de la transparencia para que los sesgos de las máquinas no vengan de los sesgos de las personas (antes nos hemos referido al machine learning que aprende de la gente y que va a tener una información u otra según quien opine).

Internet es más que nunca una fuente inagotable de oportunidades y un recordatorio constante de que "la información es poder" (Gilardoni, 2013).

\section{Bibliografía}

Angwin, Julia; Larson, Jeff; Mattu, Surya; Kirchner, Lauren (2016). "Machine bias: There's software used across the country to predict future criminals. And it's biased against blacks". ProPublica, May 23.

https://www.propublica.org/article/machine-bias-riskassessments-in-criminal-sentencing

Botsman, Rachel (2012). “La moneda de la nueva economía es la confianza". TED talks, junio.

https://www.ted.com/talks/rachel_botsman_the_currency of_the_new_economy_is_trust?language $=e s$

Brusilovsky, Peter (1996). "Methods and techniques of adaptive hypermedia". User modeling and user-adapted in- teraction, v. 6, n. 2, pp. 87-129.

https://doi.org/10.1007/BF00143964

Bullas, Jeff (2016). "10 top social media marketing trends to look out for in 2017". Art+marketing, Sept. 19.

https://medium.com/art-marketing/10-top-social-media-marketingtrends-to-look-out-for-in-2017-95e4b2c4e9fb\#.te4zehrnz

Capgemini Consulting (2012). The evolution of emergency preparedness and response capabilities in the oil and gas industry. Emergency preparedness and response survey. https://goo.gl/Yve7NT

Carat (2015). Carat's 10 trends for 2016, 17 Dec. http://www.slideshare.net/NextGenerationMedia/carats10-trends-for-2016

Carson, Erin (2015). "Why virtual reality could finally mend its broken promise". TechRepublic. Cover story.

http://www.techrepublic.com/article/why-virtual-realitycould-finally-mend-its-broken-promise

Cisco (2015). Cisco visual networking index: Forecast and methodology, 2014-2019. May 27.

https://goo.gl/xoBrTA

Condliffe, Jamie (2016). “Google podría conseguir el primer ordenador cuántico funcional en 2017". MIT technology review, 5 septiembre.

http://www.technologyreview.es/informatica/51564/ google-podria-conseguir-el-primer-ordenador

Dans, Enrique (2016). “Entre personas y algoritmos, gana el algoritmo". Enrique Dans, 29 agosto.

https://www.enriquedans.com/2016/08/entre-personas-yalgoritmos-gana-el-algoritmo.html

Gilardoni, Claudia (2013). “La información ¿̇es poder?”. Infotecarios, 5 febrero.

http://www.infotecarios.com/la-informacion-es-poder

Kim, Larry (2015). "3 trends that will change the future of online advertising". WordStream. Online advertising made easy. The Wordstream blog, July 12.

http://www.wordstream.com/blog/ws/2015/05/04/futureof-online-advertising

Koetsier, John (2014). "Social media: We complain 879 million times/year (and Facebook is our top target)". VB insight, Dec. 12. http://venturebeat.com/2014/12/12/social-media-wecomplain-879-million-timesyear-and-facebook-is-our-top-target

Manson, Marshall; Whatley, James (2015). Key digital trends for 2016, Dec. 16.

http://www.slideshare.net/socialogilvy/key-digital-trendsfor-2016

Meeker, Mary (2016). Internet trends 2016. Code conference. June 1.

http://www.kpcb.com/internet-trends

Merton, Robert K. (1968). "The Matthew effect in science". Science, v. 159, n. 3810, pp. 56-63.

http://www.garfield.library.upenn.edu/merton/matthew1.pdf

RAE (2016). "Paradigma”. Diccionario de la lengua española. Edición del Tricentenario. Real Academia de la Lengua.

http://dle.rae.es/?id=RpXSRZJ 
Ros-Selva, Jaume (2015). "La fragmentación del discurso publicitario. Usuarios, omnicanalidad e influencia sobre el proceso de creación y difusión de mensajes publicitarios". Revista académica liLETRAd, n. 1, pp. 331-340.

Rosenberg, Seth (2016). How to build bots for Messenger. Facebook. Noticias para desarrolladores, 12 abril. https://developers.facebook.com/blog/post/2016/04/12/ bots-for-messenger

Rosso, Dan (2016). "International technology roadmap for semiconductors examines next 15 years of chip innovation". Semiconductor Industry Association (SIA). News, July 8. https://goo.gl/JT1HjX

The economist (2013). "The rise of the sharing economy". The economist, March 9. https://goo.gl/isiDo
Weber, Rolf H. (2013). "Internet of things - Governance quo vadis?". Computer law \& security review, v. 29, n. 4, pp. 341347.

https://www.researchgate.net/publication/259123292_ Internet_of_things_-_Governance_quo_vadis http://dx.doi.org/10.1016/j.clsr.2013.05.010

Westerman, George; Bonnet, Didier; McAfee, Andrew (2014). "The nine elements of digital transformation". MIT Sloan management review, January 7.

http://sloanreview.mit.edu/article/the-nine-elements-ofdigital-transformation

Wu, Tim (2010). Master switch: The rise and fall of information empires. New York: Knopf. ISBN: 0307269930

YouTube (2015). Statistics, March.

https://www.youtube.com/yt/press/statistics.html

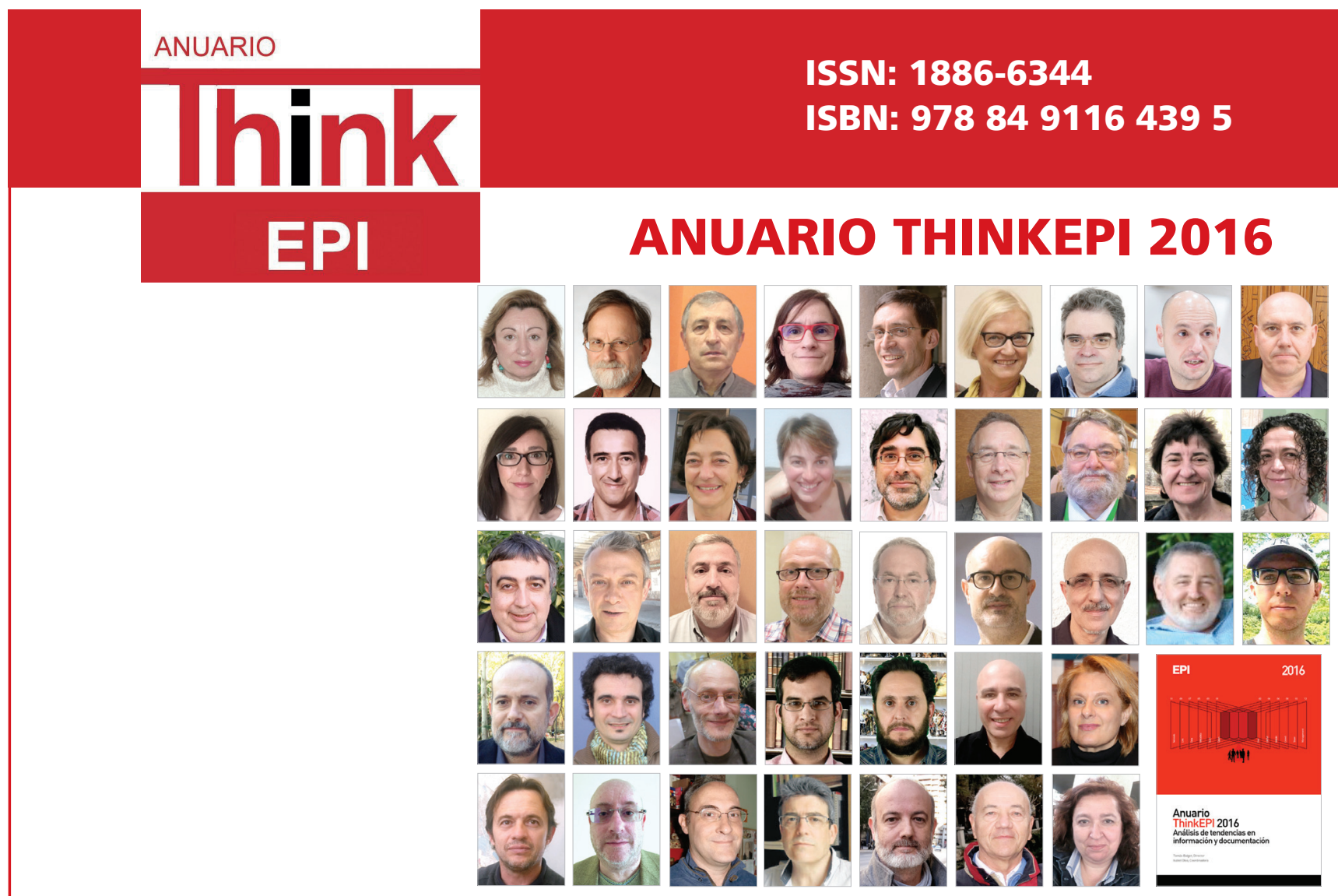

\section{PRECIOS ANUARIO THINKEPI}

Suscripción online (2007-2016)

Instituciones ...................................... $80 €$

Individuos (particulares) $48 €$

Números sueltos

Instituciones

Anuario ThinkEPI 2016 (pdf)

$55 €$

Anuario de años anteriores* $30 €$

Individuos (particulares)

Anuario ThinkEPI 2016 (pdf)............. $30 €$

Anuario de años anteriores* ............ $22 €$

*Años 2007 a 2013 disponibles en papel + pdf.

A partir de 2014 sólo disponible en pdf
Desde 2014 es posible el acceso mediante suscripción a todos los Anuarios ThinkEPI publicados hasta el momento desde el Recyt de la Fecyt

http://recyt.fecyt.es/index.php/ThinkEPI

\section{Más información:}

Isabel Olea

epi.iolea@gmail.com 